\title{
Calidad de atención integral brindada por madres cuidadoras a niños menores de 3 años, beneficiarios del Programa Nacional Cuna Más, \\ Huánuco
}

Quality of comprehensive care provided by caregiver-mothers to children under 3 years of age, beneficiaries of Cuna Más National Program, Huánuco

Yudi Adriano Huaytán'

Mely Ruiz-Aquino²

RECIBIDO: FEBRERO 022019

ACEPTADO: MAYO 282019

1 Programa de Estudios de Enfermería, egresada, Universidad de Huánuco, Huánuco, Perú. yudyadriano@gmail.com.pe, https://orcid.org/0000-0002-9584-675979

2 Magister en Salud Pública y Gestión Sanitaria, Licenciada en Enfermería, docente, Universidad de Huánuco, Huánuco, Perú. melyruizaquino@udh.edu.pe, https://orcid. org/0000-0002-8340-7898

Socialium revista científica de Ciencias Sociales, Vol 3 - No. 2, julio - diciembre 2019, pág. 56-74.

DOI https://doi.org/10.26490/uncp.sl.2019.3.2.574 


\section{Resumen}

Objetivo. Describir la calidad de atención integral brindada por madres cuidadoras a niños menores de 3 años, beneficiarios del Programa Nacional Cuna Más; Huánuco, 2019. Métodos. Fue un estudio transversal, prospectivo y observacional, con diseño descriptivo y enfoque cuantitativo. La muestra fueron 80 cuidadoras de un total de 100 cuidadoras del servicio de cuidado diurno del Programa Nacional Cuna Más, Perú. Se aplicó una guía de entrevista de las características generales y una escala de calidad de atención; esta última fue válido y fiable. Se tuvieron en consideración los aspectos éticos de la investigación, además se realizó un análisis de frecuencia a nivel descriptivo. Resultados. Se verificó la calidad de atención integral en sus tres componentes. En la dimensión aprendizaje infantil se evidenció un 70 \% (56) de calidad en un nivel alto; en la dimensión de salud infantil el 71,3 \% (57) mostró un nivel medio de calidad; y en la dimensión nutrición y alimentación, un 27,5 \% (22) evidenció baja calidad de atención. De modo global, un 52,5 \% (42) evidenció una calidad de atención integral de nivel medio, seguido de un $33,8 \%$ (27) con calidad de atención de nivel alto. Conclusión. La calidad de atención integral al niño beneficiario del servicio de cuidado diurno del Programa Nacional Cuna Más, a cargo de madres cuidadoras, fue de nivel medio; lo que evidencia la necesidad de fortalecer las competencias del cuidado integral a los niños beneficiarios y la supervisión constante por especialistas del cuidado como los profesionales de enfermería.

Palabras clave: calidad de atención, servicio de cuidado diurno, Programa Nacional Cuna Más, niños beneficiarios

\section{Abstract}

Objective:To describe the quality of comprehensive care provided by caregivermothers for children under 3 years old, beneficiaries of Cuna Más National Program, Huánuco, 2019. Methods: It was a cross-sectional, prospective and observational study, with a descriptive design and a quantitative approach. Sample comprised of 80 caregivers of a total of 100 caregivers of the day care service of Cuna Más National Program, Peru. An interview guide of general characteristics and a scale of care quality were applied, the latter was validated 
and reliable. The ethical aspects of the research were taken into consideration. A frequency analysis was carried out at the descriptive level. Results: Quality of comprehensive care in its three components. In the child learning dimension, evidenced $70 \%$ (56) of high-level quality. In the child health dimension, $71.3 \%$ (57) showed a medium level of quality. Finally, in the nutrition and feeding dimension, $27.5 \%$ (22) showed low quality of care. Overall, 52.5\% (42) evidenced a quality of comprehensive care at a medium level, followed by $33.8 \%$ (27) with a high-level quality of care. Conclusion: Quality of comprehensive care for the beneficiary child of the day care service of Cuna Más program, carried out by caregiver-mothers, was medium level. It shows the need to strengthen competencies of comprehensive care for beneficiary children and constant supervision by specialists of care such as nursing professionals.

Keywors: quality of care, day care service, Program National Cuna Más

\section{Introducción}

Todo niño y niña tiene derecho a que, desde su gestación, la sociedad promueva las condiciones para su desarrollo; lo que incluye la atención integral como un proceso dinámico durante el curso de vida, que involucra cambios en el componente físico y en el neurodesarrollo. Con este fin, se debe garantizar adecuados servicios de atención a los niñas y niños, tanto cuando estén sanos o con alguna morbilidad. Dichos servicios deben considerar aspectos de vigilancia en el momento de la atención para identificar factores de riesgo de forma oportuna, pertinente para su corrección y desarrollando acciones de prevención, promoción, tratamiento y rehabilitación (MINSA, 2014).

En este contexto, los programas o servicios orientados a promover una calidad de vida ofrecen un abanico amplio de formas de apoyo a los niños y sus familias en diferentes áreas (salud y nutrición, servicios de vacunación, promoción de la lactancia materna, suplementación nutricional, entre otros).

La Convención sobre los Derechos del Niño (CDN) es un tratado internacional que reconoce los derechos humanos de los niños y las niñas, definidos como personas menores de 18 años, y que obliga a los gobiernos a cumplirlos. 
Asimismo, promueve el enfoque de derechos y atención integral superando el enfoque de necesidades que ha marcado hasta hace muy poco la responsabilidad y acción del Estado en los temas de infancia. Se trata de asumir y atender las necesidades de los niños en cumplimiento de sus derechos (UNICEF, 2014).

En este marco, el Estado y la sociedad civil deben reconocer y garantizar la realización de estos derechos. El Estado es responsable de priorizar y formular políticas públicas que reduzcan las desigualdades y la exclusión social en las que viven y crecen nuestros niños y niñas; y de comprometerse a llevarlas a la práctica. Según el Plan de Acción de la Cumbre Mundial a favor de la Infancia "No hay causa que merezca más alta prioridad que la protección y el desarrollo del niño, de quien dependen la supervivencia, la estabilidad y el progreso de todas las naciones y, de hecho, de la civilización humana".

Podemos definir, en ese sentido, la atención integral como el conjunto de intervenciones articuladas y complementarias destinadas a asegurar el desarrollo y bienestar de niñas y niños en el marco de la necesidad de promocionar el desarrollo infantil dadas las brechas socioeconómicas existentes a nivel nacional; y la existencia de rezagos importantes en la población de menores ingresos económicos con marcadas brechas desarrollo infantil, en la dimensión motor, de lenguaje y socioemocional.

La primera infancia es, por lo tanto, una oportunidad única para el desarrollo del ser humano. Es eses sentido, a través de los programas sociales del gobierno peruano, ya se vienen trabajando el desarrollo e inclusión social, a nivel intersectorial e intergubernamental, para promover el desarrollo infantil temprano de niñas y niños menores de 3 años de edad, en zonas en situación de pobreza y pobreza extrema para superar las brechas en su desarrollo cognitivo, social, físico y emocional.

Como en todo país, la política social es clave para asegurar la existencia de un crecimiento inclusivo que contribuya al desarrollo sostenible y el ejercicio pleno de los derechos. Tal como expresa Arguedas (2014), los programas sociales se han convertido en una herramienta política y social, caracterizado por asistir directamente a una población objetivo en situación de alta vulnerabilidad económica y social, como lo son los niños. Sin embargo, muchos programas 
sociales se convierten en, instrumentos políticos del gobierno de turno.

En tanto Del Valle (2014) y Fernández (2007), explican que los programas sociales son políticas públicas orientadas a mejorar las condiciones de calidad de vida de una determinada población. Salazar (2015) y Reyes (2018), sostienen que a nivel del Perú es prioridad del Estado invertir en la infancia. En cuanto al Servicio de Cuidado Diurno (SCD) del Programa Nacional Cuna Más se evidencia un mejor estado nutricional, debido a una buena atención de calidad en la alimentación nutricional, mejor estado de salud y consumo de micronutrientes, teniendo en cuenta la asistencia continua y la permanencia mayor a 6 meses de los niños beneficiarios. Sin embargo, se requiere una renovada Política Nacional de Desarrollo e Inclusión Social (PNDIS), con una mirada de largo plazo, que permita enfrentar la exclusión social de manera más integral y asegurar, de este modo, el cumplimiento de los derechos sociales y económicos de todas y todos (MIDIS, 2019).

Entre el $77 \%$ y el $93 \%$ de usuarios de los programas sociales que tiene a su cargo el Ministerio de Desarrollo e Inclusión Social (MIDIS) consideran que los servicios que estos brindan mejoraron su calidad de vida, según reveló un estudio de la consultora Macroconsult-Instituto Cuanto. Un 85,7\% de los usuarios consideró que el SCD del Programa Nacional Cuna Más mejoró su calidad de vida, un 77,4 \% hizo lo propio respecto del Programa Qali Warma, un $89,2 \%$ del programa Juntos, un 83,6\% del Fondo de Cooperación para el Desarrollo Social (FONCODES) y un 93,8 \% del programa Pensión 65, respectivamente. El $95 \%$ de los usuarios confía en la prestación de Cuna Más, Qali Warma, Juntos, Haku Wiñay y Pensión 65 (Agencia Peruana de Noticias Andina, 2019), los programas que fueron estudiados. En Huánuco el 43,8\% de los niños evidenciaron el control completo de crecimiento y desarrollo, dicho indicador es parte de la calidad de atención (Huacachino, 2018).

El Programa Nacional Cuna Más, programa social focalizado a cargo del MIDIS, fue creado sobre la base del ex Programa Nacional Wawa Wasi. Este programa brinda atención integral a niñas y niños menores de 3 años y madres gestantes de zonas urbanas y rurales en condiciones de pobreza y pobreza extrema. Busca mejorar el desarrollo infantil temprano para superar las brechas en el desarrollo cognitivo, social, físico y emocional de los infantes (Plataforma digital única Cuna Más, 2019). El SCD, brinda atención integral a 
niños de 6 meses a 3 años de edad que requieren atención de necesidades vinculadas a salud, nutrición, seguridad, protección, afecto, descanso, juego, aprendizaje y desarrollo de habilidades. Esta atención se brinda en los Centros Cuna Más de Cuidado Diurno, que son cogestionados entre el Estado y la comunidad; quienes brindan el servicio son madres cuidadoras, capacitadas y calificadas que garantizan una adecuada atención de las necesidades básicas de salud, nutrición, seguridad, protección, afecto, descanso, juego, aprendizaje y desarrollo de habilidades en las niñas y niños usuarios de este servicio.

Actualmente el Programa, cuenta con la autorización del Sistema Nacional de Evaluación, Acreditación y Certificación de la Calidad Educativa (SINEACE) por cinco años, como entidad certificadora de competencias en la ocupación del Cuidador(a) comunitario(a) de atención integral a niñas y niños de 0 a 36 meses", con el fin de garantizar el servicio de calidad que brinda a las niñas y niños menores de tres años.

La escasa información respecto a los resultados de los programas sociales se debe, principalmente, a la falta de estudios periódicos y longitudinales que midan el impacto en la población respecto a la calidad de atención que reciben del Estado a través de los programas sociales. La calidad de servicio y satisfacción son variables que se investigan en muchos estudios sobre gestión privada y pública, debido a la importancia que tienen para la sostenibilidad de un negocio o servicio. A nivel de programas sociales son muy pocos los estudios sobre calidad de atención integral. En igual sentido, de acuerdo a como expresan Cárdenas (2016) y Ríos (2015), la calidad de atención implica el hecho de brindar una atención integrada óptima, eficaz y eficiente para una infancia saludable; es más, la intervención en la niñez, requiere la participación de toda la sociedad para garantizar servicios de calidad enfocados a la población de mayor vulnerabilidad, como son los niños.

Asimismo, tal como señala Martínez (2017), la calidad del servicio, generalmente reclamada por los usuarios, es un problema generalizado a nivel internacional, nacional y local. Cuando se trata de los programas sociales implementados por el Estado peruano, puede estar siendo producido por la falta de una gerencia social con enfoque humano de lo público, con perspectivas de desarrollo 
social en la aplicación de las políticas sociales, innovando el enfoque hacia la creación del valor público y de inclusión social con eficiencia.

Por su parte, Cavero, Cruzado y Cuadra (2017), sostienen que, si bien el Programa Nacional Cuna Más parece tener potencial para mejorar las dimensiones cognitivas y de comunicación de los niños menores de 3 años. Por otro lado, no parece tener efectos sobre el conocimiento de las madres sobre las prácticas de cuidado infantil relacionadas a la alimentación y cuidado del menor, ni tampoco sobre nivel nutricional de los niños. Cabe señalar que, si bien el nivel nutricional de los niños no era un objetivo del programa, sin embargo, si es una condición necesaria para mejorar las dimensiones del desarrollo infantil. En el 2018 se reportaron denuncias por presuntos maltratos a los menores beneficiarios del programa, evidenciando así que se debe reforzar el proceso de selección de las madres cuidadoras de Cuna Más que son propuestas por la comunidad mediante la evaluación permanente de la calidad de atención brindada. Asimismo, se ha evidenciado que existe una débil supervisión a la madre cuidadoras por parte de los acompañantes técnicos (AT); por lo mismo que el número de los AT es reducido y por años ha existido una brecha, ya que sería necesario contar con un AT por cada comité.

En línea con lo descrito, es necesario enfatizar la importancia en la calidad de atención integral que debe brindar la madre cuidadora, procurando la optimización del aprendizaje y el desarrollo de habilidades en niños menores de 3 años. Es preciso reiterar además que la calidad de atención es relevante para lograr la satisfacción de los beneficiarios en los programas sociales. Por tanto, el objetivo de este estudio fue describir la calidad de atención integral que brindan las madres cuidadoras a niños menores de 3 años del Programa Nacional Cuna Más en la provincia de Huánuco, Perú.

\section{Método}

Tipo de estudio

Se realizó un estudio transversal, prospectivo, con diseño descriptivo y con enfoque cuantitativo. 


\section{Población y muestra}

La población fue un total de 100 cuidadoras que, según el reporte del Programa Nacional Cuna Más, Huánuco, del 2019, trabajaban en los sectores de Pomares, Jactay, Moras, Loma Blanca y Pillco Marca, dentro de la jurisdicción del distrito de Huánuco. La muestra fue seleccionada probabilísticamente, siendo 80 madres cuidadoras del Programa Nacional Cuna Más. Se incluyeron a las cuidadoras responsables directas del cuidado de los niños y, cuidadoras que tuvieran un tiempo de servicio mayor a 3 meses y que aceptaron firmar el consentimiento informado.

\section{Recolección de datos}

La variable principal del estudio fue la calidad de atención integral, medida con la escala de la calidad de atención integral, constituida por tres dimensiones (salud infantil, nutrición y aprendizaje) y 23 ítems, cuyos autores fueron Duro Elena y Alva García; posteriormente esta escala fue modificada por las autoras de esta investigación. También, se incluyeron variables sociodemográficas y laborales (edad, genero, estado civil, grado de escolaridad, tiempo de servicio y tenencia de profesión).

Las técnicas usadas fueron la entrevista individualizada y la observación, las mismas que fueron aplicadas en el periodo de abril a junio del 2019. Los instrumentos se validaron a nivel cualitativo por juico de jueces y expertos, siendo estos, personas que habían realizado investigaciones previas relacionadas a programas sociales, personas cuya actividad laboral está vinculada al tema o expertos en metodología de la investigación y validación de instrumentos. Asimismo, se hizo la validación racional y la validación por aproximación a la población. A nivel cuantitativo, la confiabilidad de la escala de la calidad de atención fue mediante el alfa de Crombach, obteniéndose un valor de 0,96; lo que indicó una alta confiabilidad.

Para garantizar la calidad del llenado de los instrumentos, tres encuestadoras debidamente capacitadas aplicaron el instrumento teniendo en cuenta ciertas recomendaciones (saludar y presentarse, explicar el objetivo de la encuesta, obtener la aceptación del consentimiento informado y en enfatizar que la encuesta es anónima).

Para ejecutar la investigación se realizaron los trámites administrativos necesarios a través de una carta de autorización dirigida a la Oficina de Coordinación Territorial de Cuna Más de Huánuco para obtener el permiso de poder realizar la investigación. Previo a la aplicación de los instrumentos de recolección de datos, se solicitó el consentimiento informado a la muestra 
en estudio; además, se consideraron los principios éticos como beneficencia, no maleficencia, justicia, autonomía, veracidad y fidelidad.

Análisis estadístico

Se realizó el análisis descriptivo usando como medidas de resumen los porcentajes para variables cualitativas. Los resultados de la variable calidad de atención integral se presentan agrupados en tres categorías: alta, media y baja. Para el procesamiento de datos, se utilizó el paquete estadístico Statistical Package for the Social Sciences para Windows, versión 21.0.

\section{Resultados}

Al analizar las características demográficas, se halló que la edad promedio de las cuidadoras fue de 33 años; más de la mitad de las madres cuidadoras [53,8 \% (43)] fueron adultas jóvenes, seguido del 36,3\% (29) de cuidadoras pertenecientes al grupo de las adultas medias y a diferencia del $10 \%$ (8) que fueron adultas maduras. El estado civil de las cuidadoras en un $42,5 \%$ (34) fueron convivientes, seguido del 28,7 \% (23) con condición de solteras. En cuanto al grado de escolaridad de las cuidadoras en estudio, el $35 \%$ (28), tuvieron secundaria incompleta, seguido de un $25 \%$ (20) con grado de secundaria completa y solo el 6,3 \% (5) tuvieron el grado superior incompleta. Respecto a las características laborales de las cuidadoras, el $72,5 \%$ (58) venia laborando en promedio de un año. En cuanto a la profesión, se evidenció que solo el 8,8 $\%$ (7) fueron profesionales técnicos en enfermería, contabilidad y laboratorio; frente a la mayoría de las cuidadoras quienes no tienen profesión técnica o universitaria [91,3\% (73)] (ver tabla 1). 
Tabla 1

Características sociodemográficas y laborales de las madres cuidadoras en el servicio de cuidado diurno del Programa Nacional Cuna Más, Huánuco, 2019.

\begin{tabular}{|c|c|c|}
\hline \multirow[t]{2}{*}{ Características sociodemográficas y laborales } & \multicolumn{2}{|c|}{$\mathrm{n}=80$} \\
\hline & fi & $\%$ \\
\hline \multicolumn{3}{|l|}{ Grupo etario } \\
\hline Adulta joven (20-39) & 58 & 72,5 \\
\hline Adulta media (40-49) & 16 & 20,0 \\
\hline Adulta madura (50-59) & 6 & 7,5 \\
\hline \multicolumn{3}{|l|}{ Estado civil } \\
\hline Soltera & 23 & 28,8 \\
\hline Casada & 17 & 21,3 \\
\hline Conviviente & 34 & 42,5 \\
\hline Viuda & 1 & 1,3 \\
\hline Divorciada & 5 & 6,3 \\
\hline \multicolumn{3}{|l|}{ Grado de escolaridad } \\
\hline Primaria incompleta & 6 & 7,5 \\
\hline Primaria completa & 14 & 17,5 \\
\hline Secundaria incompleta & 20 & 25,0 \\
\hline Secundaria completa & 28 & 35,0 \\
\hline Superior incompleta & 7 & 8,8 \\
\hline Superior completa & 5 & 6,3 \\
\hline \multicolumn{3}{|l|}{ Tiempo de servicio } \\
\hline 1 año & 58 & 72,5 \\
\hline De 5 a 10 años & 9 & 11,3 \\
\hline De 11 a 15 años & 8 & 10,0 \\
\hline Más de 15 años & 5 & 6,3 \\
\hline \multicolumn{3}{|l|}{ Profesión técnica o universitaria } \\
\hline Sí & 7 & 8,8 \\
\hline No & 73 & 91,3 \\
\hline \multicolumn{3}{|l|}{ Tipo de profesión } \\
\hline Técnico en enfermería & 3 & 3,8 \\
\hline Técnico en contabilidad & 2 & 2,5 \\
\hline Técnico de laboratorio & 1 & 1,3 \\
\hline Profesora & 1 & 1,3 \\
\hline
\end{tabular}

Nota. Guía de entrevista de las características generales de la muestra.

De la observación a las madres cuidadoras participantes del estudio. la varia- 
ble calidad de atención integral según sus tres componentes, se evidenció: el $70 \%$ (56) con una calidad de nivel alta de atención integral en la dimensión aprendizaje infantil; el 71,3 \% (57) tuvo una calidad de nivel media en la dimensión de salud infantil; un 27,5 \% (22) evidenció una baja calidad de atención en la dimensión nutrición y alimentación (ver tabla 2).

Tabla 2

Descripción de la calidad de atención según componentes, percibida por las madres cuidadoras del servicio de cuidado diurno del Programa Nacional Cuna Más, Huánuco, 2019.

\begin{tabular}{lccccc}
\hline \multirow{2}{*}{ Dimensiones } & \multicolumn{4}{c}{$\mathbf{n = 8 0}$} \\
\cline { 2 - 6 } & \multicolumn{4}{c}{ Medio } & \multicolumn{1}{c}{ Bajo } \\
\cline { 2 - 6 } & \% & Fi & $\%$ & fi & $\%$ \\
\hline Salud infantil & 57 & 6 & 7,5 \\
$\begin{array}{l}\text { Nutrición y alimenta- } \\
\text { ción }\end{array}$ & 44 & 22 & \\
Aprendizaje infantil & - & & & & \\
\hline
\end{tabular}

Nota. Escala de la atención integral en el servicio del cuidado diurno del Programa Nacional Cuna Más.

Al analizar de forma global la variable calidad de atención integral que brindaron las madres cuidadoras, se halló una significativa proporción, de un 52, $5 \%$ (42), que brindaba una calidad de atención integral de nivel medio, seguida de un 33,8 \% (27) que brindaba una calidad de nivel alto (ver tabla 3).

Tabla 3

Nivel de calidad de atención brindada por las madres cuidadoras en el servicio de cuidado diurno del Programa Nacional Cuna Más, Huánuco, 2019.

\begin{tabular}{lcc}
\hline \multirow{2}{*}{ Nivel de calidad de atención } & \multicolumn{2}{c}{$\mathbf{n}=\mathbf{8 0}$} \\
\cline { 2 - 3 } & $\mathbf{f i}$ & $\mathbf{\%}$ \\
\hline Alto & 27 & 33,8 \\
Medio & 42 & 52,5 \\
Bajo & 11 & 13,8 \\
\hline
\end{tabular}

Nota. Escala de la atención integral en el servicio del cuidado diurno del Programa Nacional Cuna Más. 
Finalmente, se comparó la variable calidad de atención con características sociodemográficas y laborales de las madres cuidadoras en el SCD del Programa Nacional Cuna Más. Aquí se evidenció que las madres cuidadoras adultas jóvenes (20 a 39 años) fueron las que brindaban una mejor calidad de atención integral [25\% (20) con calidad de nivel media y 37,5\% (30) con calidad de nivel alta]. Respecto al estado civil, las madres cuidadoras con condición de convivientes fueron las que evidenciaron una mejor calidad de atención (media 21,3\% (17) y alta 13,8 \% (11). En lo que respecta al grado de escolaridad, fueron las madres cuidadoras con el grado de secundaria las que brindaban mejor calidad de atención. Según el tiempo de servicio las cuidadoras que venían laborando por un año tuvieron mejor calidad de atención integral [23,85 (19) con calidad de nivel alta y 37, 5 \% (30) con calidad de nivel media]. En última instancia, al analizar la calidad de atención integral según la tenencia de alguna profesión técnica o universitaria, se halló que las madres cuidadoras sin profesión en un 50 \% (40) evidenciaron calidad de atención de nivel medio, seguido de un 28,8 \% (23) que evidenció una calidad de nivel alta (ver tabla 4).

\section{Tabla 4}

Variable calidad de atención según características sociodemográficas y laborales de las madres cuidadoras en el servicio de cuidado diurno del Programa Nacional Cuna Más, Huánuco, 2019.

\begin{tabular}{|c|c|c|c|c|c|c|}
\hline \multirow{4}{*}{$\begin{array}{l}\text { Carac- } \\
\text { terísticas } \\
\text { sociodemo- } \\
\text { gráficas y } \\
\text { laborales }\end{array}$} & \multirow{4}{*}{ Variables } & \multicolumn{5}{|c|}{$\mathrm{n}=80$} \\
\hline & & \multicolumn{5}{|c|}{ Calidad de atención } \\
\hline & & \multirow{2}{*}{\begin{tabular}{|r|} 
Alto \\
$\%$
\end{tabular}} & \multicolumn{2}{|c|}{ Medio } & \multicolumn{2}{|c|}{ Bajo } \\
\hline & & & fi & $\%$ & $\mathrm{fi}$ & $\%$ \\
\hline & $\begin{array}{l}\text { Adulto joven } \\
(20-39)\end{array}$ & & & & 8 & \\
\hline \multirow[t]{2}{*}{$\begin{array}{l}\text { Grupo eta- } \\
\text { rio }\end{array}$} & $\begin{array}{l}\text { Adulto me- } \\
\text { dio (40-49) }\end{array}$ & & 9 & & 1 & \\
\hline & $\begin{array}{l}\text { Adulto ma- } \\
\text { duro (50-59) }\end{array}$ & & 3 & 3,8 & 2 & \\
\hline
\end{tabular}




\begin{tabular}{|c|c|c|c|c|}
\hline \multirow{5}{*}{ Estado civil } & Soltera & & & 2 \\
\hline & Casada & & & 2 \\
\hline & Conviviente & & & 6 \\
\hline & Viuda & 0 & 0,0 & 0 \\
\hline & Divorciada & 2 & 2,5 & 1 \\
\hline \multirow{6}{*}{$\begin{array}{l}\text { Grado de } \\
\text { escolaridad }\end{array}$} & $\begin{array}{l}\text { Primaria in- } \\
\text { completa }\end{array}$ & 3 & 3,8 & 1 \\
\hline & $\begin{array}{l}\text { Primaria } \\
\text { completa }\end{array}$ & 6 & 7,5 & 2 \\
\hline & $\begin{array}{l}\text { Secundaria } \\
\text { incompleta }\end{array}$ & & & 1 \\
\hline & $\begin{array}{l}\text { Secundaria } \\
\text { completa }\end{array}$ & & & 5 \\
\hline & $\begin{array}{l}\text { Superior in- } \\
\text { completa }\end{array}$ & 3 & 3,8 & 1 \\
\hline & $\begin{array}{l}\text { Superior } \\
\text { completa }\end{array}$ & 2 & 2,5 & 1 \\
\hline \multirow{4}{*}{$\begin{array}{l}\text { Tiempo de } \\
\text { servicio }\end{array}$} & 1 año & & & 9 \\
\hline & $\begin{array}{l}\text { De } 5 \text { a } 10 \\
\text { años }\end{array}$ & 4 & 5,0 & 1 \\
\hline & $\begin{array}{l}\text { De } 11 \text { a } 15 \\
\text { años }\end{array}$ & 4 & 5,0 & 1 \\
\hline & $\begin{array}{l}\text { Más de } 15 \\
\text { años }\end{array}$ & 4 & 5,0 & 0 \\
\hline $\begin{array}{l}\text { Profesión } \\
\text { técnica o } \\
\text { universitaria }\end{array}$ & $\begin{array}{l}\mathrm{Si} \\
\mathrm{No}\end{array}$ & 2 & 2,5 & 1 \\
\hline
\end{tabular}

Nota. Guía de entrevista de las características generales de la muestra y escala de la atención integral en el servicio del cuidado diurno del Programa Nacional Cuna Más.

\section{DISCUSIÓN}

Durante los primeros años de vida, los niños se desarrollan a partir de la interacción con el medio físico y social que los rodea; es por ello que la primera infancia es considerada como la ventana de oportunidad más importante para sentar las bases del desarrollo futuro de los niños. En este período, tal como lo explica Tarrillo (2017), el aprendizaje es más eficiente. Y, por lo general, se sientan las bases del desarrollo físico, psíquico y social del niño. Las intervenciones en la primera infancia son intervenciones que deben desarrollar los gobiernos, tal como está consignado en la Declaración de los Derechos de los Niños (Martínez, 2014). La inversión en la primera infancia, en general, y en 
el Desarrollo Infantil Temprano (DIT), en particular, se justifica apelando a distintos tipos de argumentos: el reconocimiento del derecho de los niños a vivir un presente pleno y acceder a oportunidades para el futuro; la identificación de los primeros años de vida como un momento crucial para el desarrollo de capacidades y competencias cognitivas, afectivas y sociales; y las altas tasas de retorno que conlleva la inversión social en la primera infancia.

Por su parte, Mello, Henrique, Pancieri, Veríssimo, Tonete y Malone (2014), explican que para la promoción de la salud infantil es esencial la comprensión de sus peculiaridades, así como las condiciones ambientales favorables para su desarrollo. La comprensión de los cuidadores acerca de las características y necesidades de los niños, como resultado de su proceso de desarrollo, facilita un desarrollo integral, ya que los cuidados diarios son los espacios de promoción del desarrollo infantil. Las intervenciones en la infancia deben involucrar además a las familias, cuidadores profesionales como profesionales de enfermería y otros grupos de educación.

El modelo ecológico de Bronfenbrenner considera que el desarrollo humano es el proceso por el que la persona adquiere una concepción más amplia, diferenciada y válida de su medio de vida (entorno ecológico); se hace más motivada y capaz de realizar actividades que revelen las propiedades del mismo, de mantenerlas o reestructurarlas en su forma y en su contenido, en niveles de complejidad parecida o superior. A cada ámbito en que el niño está presente Bronfenbrenner lo denomina microsistema. La transición ecológica primordial es, evidentemente el nacimiento, no sólo para la criatura sino igualmente para los padres (Molina, Cordero, Silva 2008). Sin embargo, no todos los niños están expuestos a experiencias tempranas que les permiten alcanzar su máximo potencial de desarrollo. Precisamente, debido a ello, los países cuentan con políticas y programas específicamente orientados a promover el desarrollo infantil temprano. El Perú no es ajeno a esta situación y existe el reconocimiento de la primera infancia como una prioridad del Estado y las políticas públicas en varios documentos de política vigentes (MIDIS, 2017).

El MIDIS (2012), como ente rector de la política de desarrollo e inclusión social, gestiona y ejecuta programas sociales que aseguran una intervención coordinada y eficaz en la dotación de bienes y servicios a la población en proceso de inclusión. El Programa Nacional Cuna Más es un programa social focaliza- 
do a cargo del Ministerio de Desarrollo e Inclusión Social (MIDIS) y define el desarrollo infantil de manera multidimensional, abarcando las dimensiones física, cognitiva y socioemocional del niño o niña. El objetivo de su intervención es mejorar el desarrollo infantil de niños menores de 3 años de edad en zonas de pobreza y pobreza extrema (Bravo, Reyes, Acurio y Velásquez, 2014).

A través del SCD, del Programa Nacional Cuna Más cubre las necesidades básicas de salud, nutrición, seguridad, protección, afecto, descanso, juego, aprendizaje y desarrollo de habilidades de niños menores de 3 años. Para mejorar lo descrito, el MIDIS viene asumiendo el proceso de reorganizar los programas adscritos a dicho programa, a fin de lograr que su conformación, organización y estructura orgánica sean las idóneas para ejecutar, en el marco de los principios y valores antes indicados, las prestaciones y actividades de carácter temporal que se encuentran a su cargo.

En bases a estas premisas, la presente investigación tuvo como resultado una alta proporción de calidad de atención integral de nivel medio. Este resultado quizá se fundamente en la complejidad que resulta medir la calidad de atención integral, en la heterogeneidad de la calidad del servicio, en la alta rotación que existe de las madres cuidadoras; lo cual amerita continuar con más estudios. Resulta reseñable la congruencia de nuestros hallazgos con el estudio de Carmen y Sosa (2018), quien, a nivel de un establecimiento de salud, en el programa de crecimiento y desarrollo del niño, halló que el $38 \%$ tuvo una percepción de calidad de atención media y el resto se distribuye en $34 \%$ con una percepción de calidad de atención de nivel baja.

Por su parte, el estudio de Martínez (2017) halló que la percepción de los colaboradores acerca de la calidad de servicio en el Programa Nacional Cuna Más en el expresaba en un $23,3 \%$ de la muestra "siempre ocurre", en un 51,7 \% "a veces ocurre" y en un $25 \%$ "nunca ocurre".

En otro estudio próximo a nuestros resultados, a nivel del Programa Juntos, Quispe (2018) halló que la calificación de la calidad de atención en la variable "trato de los trabajadores del programa Juntos hacia los beneficiarios", un $47 \%$ respondieron que el trato recibido por parte del personal que labora en el Programa Juntos es "regular", un $44 \%$ menciono que el trato recibido por parte del personal que labora es "bueno", un $6 \%$ menciono que es "muy bue- 
no" y, finalmente, un 3 \% dio a conocer que recibió un "mal trato" por parte del personal.

Por otro lado, nuestro resultado es distante con el estudio del propio MIDIS (2017), quienes evaluaron el impacto del SCD en el desarrollo de los niños, entre 30 y 36 meses, a partir del análisis de bases de datos secundarias, como la Encuesta Demográfica y de Salud Familiar (ENDES). En ese sentido, evaluaron las tres dimensiones del desarrollo infantil que son parte también de la calidad de atención integral que se abordan en nuestra investigación. Sus resultados encuentran un impacto positivo y significativo de la asistencia a un SCD Cuna Más sobre el desarrollo infantil, aunque al mismo tiempo reconocieron algunas limitaciones sobre los resultados, toda vez que trabajaron con datos provenientes de fuentes secundarias.

Finalmente, se reconoce que medir la calidad de atención integral es un tema complejo y muy heterogéneo. Se tuvo limitaciones para discutir los resultados dado que no se cuenta con estudios realizados sobre calidad de atención integral a nivel del Programa Nacional Cuna Más. De otra parte, se recomienda fortalecer las capacidades de las madres cuidadoras en la atención integral de los niños, en cada uno de sus componentes, desarrollando estrategias, sesiones de socialización e interaprendizaje para brindar una mejor calidad en la atención de los niños que acudan al SCD del Programa. Asimismo, es necesaria la supervisión constante a las madres cuidadoras por especialistas del cuidado, como los profesionales de enfermería que, conforme a la ley, tienen entre sus funciones, la gestión del cuidado, el control de crecimiento y desarrollo del niño, la estimulación temprana del niño, la promoción de la salud y la prevención de enfermedades. De igual forma, se recomienda desarrollar estudios con enfoque mixto sobre la calidad de atención integral, con evaluaciones longitudinales y aplicando el modelo SERVQUAL. También, es necesario estudiar variables como la satisfacción de los padres de familia frente a los cuidados que se brindan en los SCD del Programa Nacional Cuna Más, a fin de que nuevos aspectos puedan ser considerados en la mejora de la calidad integral del mismo. 


\section{Conclusión}

La calidad de atención integral al niño beneficiario del servicio de cuidado diurno del Programa Nacional Cuna Más, a cargo de madres cuidadoras, fue de nivel medio; lo que evidencia la necesidad de fortalecer las competencias del cuidado integral a los niños beneficiarios y la supervisión constante por especialistas del cuidado como los profesionales de enfermería.

\section{REFERENCIAS BIBLIOGRÁFICAS.}

Agencia Peruana de Noticias Andina. (21 de julio de 2019). Entre $77 \%$ y $93 \%$ de usuarios de programas sociales considera que mejoró su calidad de vida. Lima: Agencia Peruana de Noticias. Recuperado de https://andina.pe/agencia/noticia-entre-77-y-93-usuarios-programas-sociales-considera-mejoro-su-calidad-vida-622356.aspx

Arguedas, M. (2015). Calidad de Servicio y Satisfacción de usuarios del Programa Nacional Vida Digna, Ministerio de la Mujer y Poblaciones Vulnerables Lima, 2014 (tesis de posgrado). Universidad César Vallejo, Lima, Perú.

Bernal, R. (2014). Diagnóstico y recomendaciones para la atención de calidad a la primera infancia en Colombia. Recuperado de https://www.repository. fedesarrollo.org.co/bitstream/handle/11445/148/CDF_No_51_Abril_2014. pdf?sequence=3\&isAllowed $=y$

Bravo, F., Reyes, M., Acurio, C y Velásquez, D. (2014). Informe de las inspecciones realizadas a los servicios de alimentación diurno del programa nacional cuna más, en las sedes Caylloma - Arequipa, Ayacucho y Cajamarca durante el ejercicio 2013. Bol. Inst. Nac. Salud, 20(3-4), 40-9.

Cárdenas, H. (2016). La Educación Y Atención Integral Para El Desarrollo De La Niñez Menor De 3 Años En Costa Rica: Una Deuda Del Estado Costarricense. Revista Electrónica "Actualidades Investigativas en Educación", 16(1), 1-22.

Carmen, V.J. y Sosa, K.J. (2018). Satisfacción de las madres que acuden al servicio de crecimiento y desarrollo y percepción de la calidad de atención de enfermería en el centro de Salud Cooperativa Universal (tesis de pregrado). Universidad Privada Norbert Wiener, Lima, Perú.

Cavero-Arguedas, D., Cruzado de la Vega, V. y Cuadra-Carrasco, G. (2017). Los efectos de los programas sociales en la salud de la población en condi- 
ción de pobreza: evidencias a partir de las evaluaciones de impacto del presupuesto por resultados a programas sociales en Perú. Revista Peruana de Medicina Experimental y Salud Pública, 34(3), 528-537. Recuperado de https://www.redalyc.org/articulo.oa?id=363/36353391021

Huacachino, Y. (2018). Cumplimiento de control de crecimiento y desarrollo y salud general en niños menores de un año. Aclas Las Moras - Huánuco 2017 (tesis de pregrado). Universidad de Huánuco, Huánuco, Perú.

Martínez, V. (2019). La gerencia social y calidad del servicio en el programa nacional Cuna Mas Ancash, 2017. (tesis postgrado). Universidad Nacional Santiago Antunez de Mayolo, Huaraz, Perú.

Martínez, J. (2014). Desarrollo infantil: una revisión. Investigaciones Andina, 16(29), 1118-1137. Recuperado de https://www.redalyc.org/articulo. oa? id=2390/239031 678009

Mello, D.F., Henrique, N.C.P., Pancieri, L., Veríssimo, M.L.Ó.R., Tonete, V.L.P. y Malone, M. (2014). Childs afety from the perspective of essential needs. Rev. Latino-Am. EnfermagemJuly, 22(4): 604-10.

Ministerio de Desarrollo e Inclusión Social. (2019). Política Nacional de Desarrollo e Inclusión Social a 2030. Recuperado de http://sdv.midis.gob.pe/Sis_Consulta_PNDIS/archivos/PNDIS-borrador-completo.pdf

Ministerio de Desarrollo e Inclusión Social. (2017). Evaluación de Impacto del Servicio de Cuidado Diurno del Programa Nacional Cuna Más - Resultados finales. Recuperado de http://evidencia.midis.gob.pe/wp-content/ uploads/2018/05/Informe_Final_9-1.pdf

Ministerio de Salud. (2011). Modelo de Atención Integral en Salud basado en Familia y Comunidad. Recuperado de http://bvs.minsa.gob.pe/local/MINSA/1617. pdf

Molina M.H., Cordero, V.M. y Silva,V. V. (2008). De la sobrevida al desarrollo integral de la infancia: Pasos en el desarrollo del sistema de protección integral a la infancia. Rev Chil pediatría, 9(1): 11-7.

Noceti, M. (2005). "Organizaciones fuertes". Presencia y decisión en el devenir de las políticas públicas dirigidas a niños en riesgo social en la provincia de buenos aires. Revista electrónica de estudios latinoamericanos, 3(11), 17-29.

Recuperado de http://www.redalyc.org/articulo.oa? id=496451226002. 
Plataforma digital única del Estado Peruano. (2019). Acceder a servicios para niñas y niños - Programa Cuna Más. Recuperado de https://www.gob.pe/580-ministerio-de-desarrollo-e-inclusion-social-acceder-a-servicios-para-ninas-y-ninos-programa-cuna-ma

Quispe, C. (2018). Percepción del impacto del Programa Juntos, en los beneficiarios del distrito de San Juan de Tarucani, de la provincia de Arequipa, 2018 (tesis de pregrado). Universidad Nacional De San Agustín, Arequipa, Perú.

Reyes, J. (2018). Influencia del servicio de cuidado diurno del Programa Nacional Cuna Mas para mejorar el desarrollo infantil en los niños y niñas usuarios del centro poblado el Milagros del distrito de Huanchaca año 2017 (tesis de postgrado). Universidad Nacional de Trujillo, Trujillo, Perú.

Ríos Montalván, D (2015). La participación familiar para el desarrollo integral de los niños y niñas del programa "CNH" (Creciendo con Nuestros Hijos)" de "La Unidad San Agustín - Motupe" de la ciudad de Loja. (Tesis de pregrado). Universidad Nacional De Loja, Loja, Ecuador.

Salazar, N. (2015). La atención integral en el desarrollo infantil de los niños y niñas menores de 3 años del Wawa Wasi Institucional - Sunarp. (Tesis de postgrado). Universidad Cesar Vallejo, Lima, Perú.

Tarrillo, W. (2017). Evaluación del impacto del programa nacional cuna más en el desarrollo de los niños egresados del servicio de acompañamiento a familias en el Distrito San Ignacio - 2017 (Tesis de postgrado). Universidad Nacional de Cajamarca, Cajamarca, Perú.

UNICEF. (2014). Convención de los Derechos del Niño. Recuperado de https://www. unicef.es/publicacion/convencion-sobre-los-derechos-del-nino 